\title{
A Note on Footnotes and Style
}

No attempt has been made to provide uniformity of editorial style in the documents and writings in this collection. Instead the style in which the particular extract originally appeared has been retained.

The footnotes in the selected extracts have been retained wherever possible. Where, however, a footnote is unrelated to the present study or refers to a passage in the publication which is not included, it has been omitted. In such a case the footnotes have not been renumbered with the result that there are occasionally gaps in the numbering of footnotes.

Footnotes to the selected extracts appear in numerals. In order to distinguish footnotes in the editor's commentary from those in the selected writings, the former are indicated by letters of the alphabet. In the more lengthy chapters (for example, Chapter 8) it has been necessary to repeat the letters of the alphabet. In such a case, where reference is made to a previous footnote $(n$.) and there are several such notes identified by the same letter, the page on which the note appears is also provided, for example, "See Smith, n. c (above p. 100)." (An example of such a case is note "a" on page 357.) 